\title{
CONSTRUCTION OF A SMOOTHED DEA FRONTIER
}

\author{
João Carlos Correia Baptista Soares de Mello* \\ Production Engineering Department \\ Fluminense Federal University \\ Niterói - RJ \\ E-mail: gmajcsm@vm.uff.br
}

Marcos Pereira Estellita Lins

Production Engineering Program

Federal University of Rio de Janeiro

Rio de Janeiro - RJ

E-mail: lins@pep.ufrj.br

Eliane Gonçalves Gomes

Satellite Monitoring Research Center

Brazilian Agricultural Research Corporation (Embrapa)

Campinas - SP

E-mail: eliane@cnpm.embrapa.br

* Corresponding author/autor para quem as correspondências devem ser encaminhadas

Received September 2001; accepted October 2002 after one revision.

\begin{abstract}
It is known that the DEA multipliers model does not allow a unique solution for the weights. This is due to the absence of unique derivatives in the extreme-efficient points, which is a consequence of the piecewise linear nature of the frontier. In this paper we propose a method to solve this problem, consisting of changing the original DEA frontier for a new one, smooth (with continuous derivatives at every point) and closest to the original frontier. We present the theoretical development for the general case, exemplified with the particular case of the BCC model with one input and one output. The 3-dimensional problem is briefly discussed. Some uses of the model are summarised, and one of them, a new Cross-Evaluation model, is presented.
\end{abstract}

Keywords: smoothed frontier, tradeoffs, Ritz method.

\section{Resumo}

O formulação dos multiplicadores para os modelos DEA não admite múltiplas soluções ótimas. Este fato pode ser interpretado no dual (modelo do envelope), como a inexistência derivadas nas DMUs extremo-eficientes, sendo esta propriedade, por seu turno, uma conseqüência da fronteira eficiente ser linear por partes. Neste artigo propõe-se substituir a fronteira original por outra, tão perto dela quanto possível, mas continuamente diferenciável. Nesta fronteira os multiplicadores sempre serão únicos para cada DMU. A teoria geral é deduzida e aplicada ao caso particular do modelo BCC com uma entrada e uma saída. A possível generalização do modelo é brevemente discutida, e são listadas algumas possíveis aplicações. É exemplificada uma das aplicações, a saber, um novo modelo de avaliação cruzada.

Palavras-chave: fronteira suavizada, razões de compensação, método de Ritz. 


\section{Introduction}

The multiplier model approach for DEA models is done via a Linear Programming Problem (LPP), which leads to multiple optimal solutions for the extreme-efficient DMUs. This multiplicity of solutions is often ignored in analyses or, sometimes a brief reference is made to it. Even so the first values found by the LPP method are used (Thanassoulis, 1993; Chilingerian, 1995).

What is sought is a method of resolution of this ambiguity in the determination of the weights, in such a form that each DMU has a single set of weights, allowing us to know the relative importance of each input or output.

To solve this problem it is necessary to interpret the weights in the dual model, that is, the envelopment model. Using the theorem of complementary slacks, it is possible to show that the weights correspond to the coefficients of the hyperplane which is tangent at each point of the efficient frontier, called the supporting hyperplane (Lins \& Angulo-Meza, 2000).

Let us consider a DEA model where $x_{i k}$ are the inputs and $y_{j k}$ the outputs; $v_{i k}^{*}$ and $u_{j k}^{*}$ are the input and output coefficients which maximise the efficiency. The equation of the hyperplane tangent to the radial projection of DMU $k$ can be written as $\sum_{j} u_{j k}^{*} y_{j k}-\sum_{i} v_{i k}^{*} x_{i k}=0$, in the CCR model, and $\sum_{j} u_{j k}^{*} y_{j k}-\sum_{i} v_{i k}^{*} x_{i k}=-u_{*}$, in the BCC model, both input oriented. The latter sentence proofs that the problem of determining a single set of weights in the multiplier model is equivalent to determining a hyperplane tangent in the envelopment model, for which is necessary to calculate the partial derivatives in all points of the frontier. The discontinuity of the derivatives at the piecewise linear frontier is an intrinsic property of the DEA methodology (Rosen et al., 1998).

The problem of choosing one solution from an infinity of optimal solutions was tackled by Doyle \& Green (1995) to deal with cross evaluation, although proposing extreme alternatives: one aggressive model and the other benevolent. Charnes et al. (1985) proposed the arbitrary use of a single value for the derivatives via a calculation of a weighted average, based on the baricenters of the competing hypersurfaces. The super-efficiency model (Andersen \& Petersen, 1993) also provides a unique set of weights for each DMU. This model has several drawbacks; for instance, it leads to an unfeasible LPP for some extremeefficient DMUs.

This paper presents a less arbitrary way to solve the problem. We replace the frontier calculated in the DEA model by another that accepts a tangent hyperplane at all its points, but which maintains the majority of the original DEA frontier properties. One property that we want to preserve is that the new frontier and the original one are determined by the same efficient DMUs. In some cases, this leads to a infeasible mathematical programming problem. In these cases, we must guarantee that the efficient DMU with the greatest distance to the smooth frontier is as closest as possible from it. This leads to a MinMax problem.

Since the only points on the frontier where there is no slope are the extreme-efficient DMUs (Charnes et al., 1988), which represent "corners" of the hypersurface, what is sought is to 
smooth these corners. In the literature we can find some smoothing problems in the framework of the non-linear programming (Koohyun \& Yong-Sik, 1998; Gal, 1992). These approaches have a different goal from the one needed in DEA.

Thus, this paper presents a methodology for the determination of a single value for the tradeoffs (and, therefore, for the multipliers), determining a new efficient frontier on which it is possible to calculate directional derivatives at all the points. In order to develop a formulation for this problem it is necessary to characterise what is understood by two frontiers close to each other, that is, a study in Functional Analysis, or the study of standard topology of functions sets.

\section{Topology of the Problem}

\subsection{Inadequacy of classical topologies}

A metric (or distance) between two elements $a$ and $b$ of a set $\boldsymbol{A}$ (where $a$ and $b$ can be points, functions, vectors, etc.) is a function $d(a, b)$ with the following properties:

1. $d(a, b) \geq 0 ; d(a, b)=0$ if and only if $a=b$

2. $d(a, b)=d(b, a)$

3. $d(a, c) \leq d(a, b)+d(b, c)$ (triangular inequality)

In the case of a DEA frontier the set $\boldsymbol{A}$ is composed of vector functions, from $\mathfrak{R}^{\mathrm{n}}$ to $\mathfrak{R}^{\mathrm{m}}$ (each coordinate of the vector is an output) with various independent variables (each one an input). Besides, the function should have certain smoothness characteristics that lead to the continuity of $1^{\text {st }}$ order derivatives. One way to assure that this continuity exists is to be sure about the existence of $2^{\text {nd }}$ order derivatives. This is somewhat constraining, but allows the usual Variational Calculus equations to be used. The theoretical considerations to be made are not affected if only output and various inputs are taken in consideration. This is the same as saying that we are going to consider the set of multi-variable functions $Z=F\left(x_{1}, x_{2}, \ldots, x_{i}, \ldots, x_{n}\right)=F(\vec{X})$ with second order partial derivatives.

Two traditional metrics for the set $\boldsymbol{A}$ are those analogous to the Euclidean and Tchebycheff ones (Prenter, 1975). Take two functions $Z_{1}=F(\vec{X})$ and $Z_{2}=G(\vec{X})$, that exists in a region R. The Euclidean and Tchebycheff metrics are defined by the following relationships:

- $d(F, G)=\int_{R}\left(Z_{1}-Z_{2}\right)^{2} d x$

- $d(F, G)=\max \left|Z_{1}-Z_{2}\right| \quad \forall \vec{X} \in \Re^{n}$

Figure 1 shows the graphical representation of these metrics. The first of them is related to the shaded area, the second is equal to the segment $\mathrm{AB}$. 


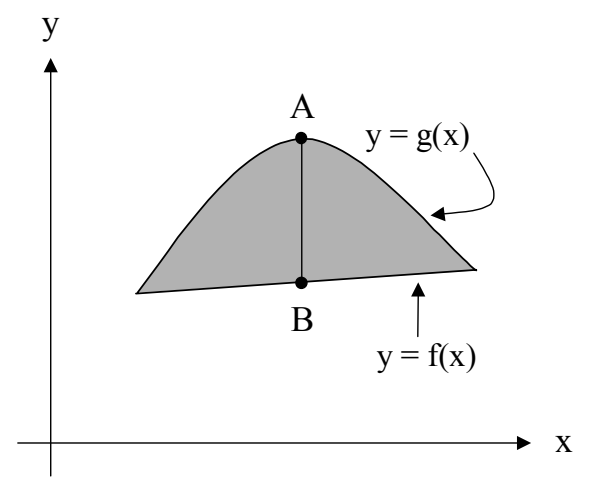

Figure 1 - Example of functional metrics.

Basically, the first metric considers that two functions are close if on average the difference between the two is small, despite some occasional high values. In the second metric, two functions are close if the largest difference between them is small. Despite their differences, the two have in common that they take only into consideration the values of the functions, leaving aside the values of their derivatives (Figure 2). However, in the present problem the important point is exactly the calculation of the derivatives. Not only should the function that represents the new frontier be close to the original frontier (for the calculated efficiencies not to be substantially different), but its slops should also have similar values to the original ones, wherever they existed.

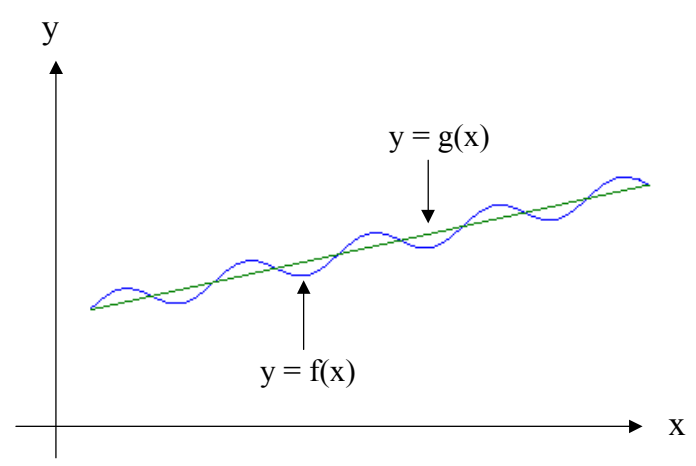

Figure 2 - Functions that are close, but with dissimilar derivatives.

To take into simultaneous consideration the distance between two functions and their derivatives, a metric must be established that takes into account the distances between the functions and between their derivatives. Using the above mentioned metrics, this can be done as follows:

- $d(F, G)=\max \left[\int_{R}\left(Z_{1}-Z_{2}\right)^{2} d x, \int_{R}\left(Z_{1}^{\prime}-Z_{2}^{\prime}\right)^{2} d x\right]$

- $d(F, G)=\max \left[\max \left|Z_{1}-Z_{2}\right|, \max \left|Z_{1}{ }^{\prime}-Z_{2}{ }^{\prime}\right|\right] \forall \vec{X} \in \mathfrak{R}^{n}$ 
This approach presents two problems. The first one is the introduction of highly complex calculations; the second one is related to the existence of points that represent extremeefficient DMUs. These are the ones of concern to this paper. However, derivatives do not exist in all frontier points. So, it does not make sense to evaluate the distances between the two derivative functions.

\subsection{Proposed topology and its properties}

Due to the problems that were pointed out in the approach described hereabove, means to determine the proximity between functions are required. These should not be the traditional and should be useful in the problem of smoothing the DEA frontier. This can be done by starting off with the case of one input and one output (which can be represented twodimensionally) to be followed by a generalisation for higher dimensions.

In the two-dimensional case, a frontier region that contains 2 consecutive efficient DMUs is a segment of a straight line. Since the segment of a straight line is the shortest arc length between two points, any other curve that connects 2 points will have a greater arc length, greater still for greater divergences from the straight line. Besides, the more it oscillates in the neighbourhood of the segment (implying the existence of derivatives whose value is substantially different from the slope of the straight line of the segment) the greater the arc length will be. Hence, if we use the differences between arc lengths to determine whether or not the smoothed frontier is located in the neighbourhood of the original frontier we are bound to consider both the function and the derivative values. Since the original frontier is made up of a set of straight segments connected in a chain, and since the straight line segment is the shortest Euclidean distance between two points, it is not necessary to calculate the arc length difference between the original frontier and the smoothed frontier. We only need to minimise the arc length of the smoothed frontier to guarantee its proximity to the original.

Before continuing with this reasoning and generalising it, some potential problems need to be looked into. For this, consider a function $f$ represented in the interval [a, b] by a certain curve. Further consider a function $g$ defined in the same interval and such that $g=f+c, c \in \Re$. It can immediately be shown that the graphical representation of $g$ is obtained from that of $f$ with a simple translation (Figure 3). Since the arc length does not vary with the translation, the difference between the arc lengths between $f$ and $g$ is zero yet the functions are not equal. Symbolically, $d(f, g)=0$ and $f \neq g$, which contradicts the first property of metrics. Thus, the arc length difference does not generate a metric.

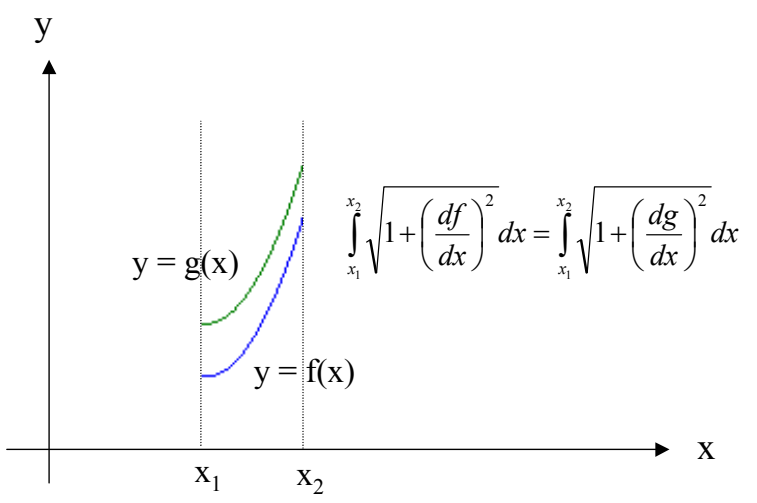

Figure 3 - Non-variance of arc length in translation. 
Even if the two functions are forced to have the same values at the interval extremes (which means that, for the same input, an extreme-efficient DMU produces the same output on the two frontiers being considered) we still do not get a metric (Figure 4). In fact, a function $h$ obtained from $f$ by symmetry about the straight line which joins the two efficient DMUs, continues to have the same arc length as $f$. Once again, we have two distinct functions with a zero difference in their arc lengths.

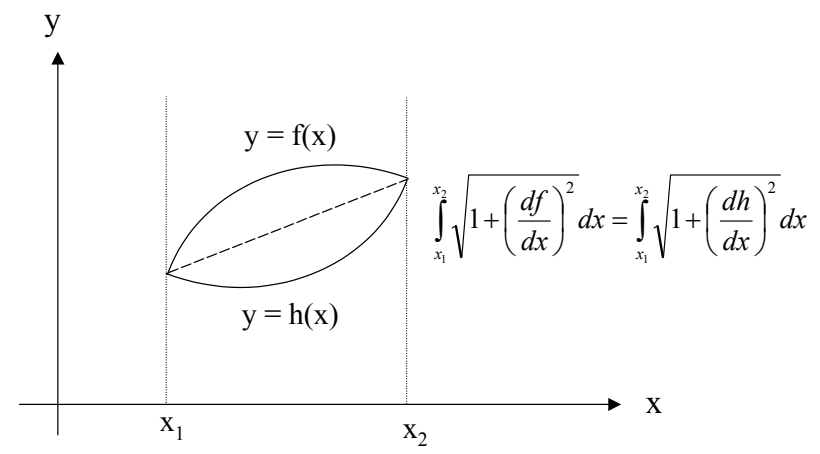

Figure 4 - Arc length equality in symmetry.

Although the arc length does not generate a metric, it does generate a non-metric topology (Lipschutz, 1968). We should not speak of distance between two frontiers, but only of proximity, or of belonging to a neighbourhood.

\subsection{Consequences of the properties of the topology proposed in DEA models}

The non-metric properties of the topology based on arc length can present some difficulties in their use in general mathematical problems. However, due to the DEA frontier characteristics, it is possible to get around these difficulties in the frontier-smoothing problem. The intent is to approximate a region of the smoothed frontier of a facet (Olesen \& Petersen, 1996) of the original frontier, which in the two-dimensional case is a segment of straight line. A segment of a straight line is the only solution to the problem of minimising the arc length between two points in an Euclidean geometry. There is only a way in which the arc length of any curve that joins two points is equal to the arc length of the straight line segment: the curve is that very straight line segment. Thus, in the specific case of DEA frontier smoothing (in which the curve distances are minimised to only-straight-line segments) we can come to the conclusion that the first condition for the definition of a metric set is obeyed. This conclusion can be generalised to higher dimensional orders. For example, in the case of two inputs and one output the arc length is replaced by the area of a surface, and the straight-line segment by a triangle.

The problem illustrated in Figure 4 determines another property for the function set resulting from the topology based on the arc length. The fact that at least two functions with the same boundary conditions and the same arc length exist means that there cannot be a neighbourhood of one which does not contain the other. In other words, the function set with topology based on arc length is not Hausdorff separable (Hausdorff, 1949). Now in this type of set it may not be possible to distinguish between two elements by their topological properties, which forces additional constraints (e.g. convexity) on the problem of arc length minimisation, so as to avoid unwanted solutions. 


\section{The Smoothed DEA Frontier}

\subsection{General formulation}

The problem of smoothing a DEA frontier with only one output consists of looking for a function which minimises the arc length (or its n-dimensional generalisation), which contains the Pareto efficient DMUs and which has second order partial derivatives at all its points. For ease of computation, the square of the arc length may be minimised without changing the result. We then have the variational problem given in (I), in which $x_{i}$ represents the inputs and $F(\vec{X})$ is the output.

To solve this problem we must know the equations of all the facets. This can be done using the method proposed by Fukuda (1993). This method grows exponentially in complexity with increase of the number of inputs and outputs.

$$
\begin{aligned}
& \min L=\int_{R}\left[1+\sum_{i}\left(\frac{\partial F}{\partial x_{i}}\right)^{2}\right] d S \\
& \text { st } \\
& F\left(\vec{X}_{j}\right)=\text { output }\left(\vec{X}_{j}\right), \quad \forall \vec{X}_{j} \in E=\{\vec{X}: \vec{X} \text { is a Pareto efficient DMU }\} \\
& \forall \vec{X}_{j}, \exists \frac{\partial F}{\partial x_{i}}
\end{aligned}
$$

It is particularly important that for the last constraint of (I) we make sure of the existence of the derivatives at the points corresponding to the extreme-efficient DMUs, because these are the points of discontinuity of the derivatives on the original frontier.

This is a problem of Variational Calculus (minimisation of a function), which can be reduced to the solution of a partial differential equation, within the given boundary conditions. The differential equation that solves a variational problem is called the "Euler-Lagrange Equation". In the present case this is $\nabla^{2} F=\sum_{i} \frac{\partial^{2} F}{\partial x_{i}^{2}}=0$ (Elsgolts, 1980).

This is the well-known n-dimensional Laplace equation (Farlow, 1993), that must be solved with suitable boundary conditions. Since the topology used does not ensure the Hausdorff separation, additional boundary conditions are necessary to characterise properly the problem. These additional conditions will be obtained from the properties of each DEA model being used. In the next section we will proof that such solution does not exist.

\subsubsection{The one output BCC model}

The existence of only one output allows us to write $O=f(I)$. To ensure that $f$ is really a function, its graphical representation cannot have vertical regions, i.e., there can be no Pareto inefficient regions on the smoothed frontier.

The fact that this is a BCC model (Banker et al., 1984) means that the frontier is convex and that requires that $\frac{\partial^{2} F}{\partial x_{i}^{2}} \leq 0$, as a corollary of the average value theorem (Mumem, 1984). This additional constraint leads to the following theorem: 


\section{Theorem}

The problem of smoothing the Pareto efficient frontier with a topology based on arc length, in a BCC model with one output, is either meaningless, or has no solution.

Proof:

a) The second derivatives are valued at zero. In this case the frontier is a hyperplane that contains all the efficient DMUs. The original frontier already has smooth characteristics, and the problem is meaningless.

b) In at least one point, one of the second derivatives is negative. Since the other second derivatives are negative or zero, their sum is negative, which contradicts the Laplace equation.

Symbolically, $\exists a_{i} \neq 0: \nabla^{2} F=\sum_{i} \frac{\partial^{2} F}{\partial x_{i}^{2}}=\sum_{i}-a_{i}^{2}<0$, and $\nabla^{2} F=0$, that is, the problem is impossible.

This theorem must be interpreted. The proposed problem is to determine the smooth frontier which best approximates the original frontier, using a topology based on arc length. To say that the problem is impossible means that there is no solution which "best approximates". Given a smooth frontier, it will always be possible to determine another (and therefore an infinity of them) that will be a better approximation to the original. The fact of not having the best approximation does not prevent the existence of good approximations, which could be calculated using variational methods, in particular an adaptation of the Ritz method (Smith, 1998).

This calculation uses an opposite approach to the Finite Elements method (Reddy, 1993), in which there is an unknown surface to be approximated by a set of polynomial functions, juxtaposed in convenient points. In the smoothing of the DEA frontier, there are polynomial functions, juxtaposed in points previously determined by the problem data, to be replaced by an unknown differentiable function.

\subsubsection{The one output CCR model}

The condition of convexity is replaced in this model by proportionality: proportional increases in the inputs cause a proportional increase in the output. That is, $F(k \cdot \vec{X})=k \cdot F(\vec{X})$, which means that $F$ has to be homogeneous in the first degree (Coelli et al., 1998). So, the function must obey the Euler's theorem for homogeneous functions: $\vec{X} \cdot \vec{\nabla} F=n F$, or, since $n=1$, $\vec{X} \cdot \vec{\nabla} F=F$. This is the additional constraint that $F$ must satisfy in the CCR model. This model will not be developed in this paper because the constraint imposed by the Euler theorem requires the development of other smoothing methods.

\subsection{Characterisation of the smoothing model for the two-dimensional DEA BCC case}

This is a model with only one input and one output and the facets are segments of straight lines. The use of a two-dimensional formulation makes Fukuda's algorithm (Fukuda, 1993) not necessary, since the facets can be determined geometrically. As seen above, there is no smooth frontier which best approximates the original, only good approximations. To determine a good approximation, we must seek to minimise not just any function, but a function chosen from within a certain family. This family of functions is called approximating functions. 
The choice of the approximating functions determines the complexity of the calculations and the quality of the solution. Quadratic approximating functions were chosen for this problem, for three main reasons:

1. Parabolas have a second derivative of constant sign, which means that they are always concave or convex. This fact allows easy application of the frontier convexity constraint.

2. According to Soares de Mello (1987), the quadratic approximating functions gives a good approximation to the solution in problems that have an exact solution, mainly when the Euler-Lagrange equation is reduced to Laplace or Poisson equations.

3. Because the approximating functions have only three coefficients to determine, the complexity of calculations is relatively low, allowing in some cases an analytical solution.

With quadratic approximating functions, if there are $n$ Pareto efficient DMUs, there will be $n-1$ straight-line segments joining these DMUs, and therefore $n-1$ functions of the form $y_{i}=a_{i} x^{2}+b_{i} x+c_{i}$. As each function has three parameters, there are $3(n-1)$ decision variables introduced into the problem of minimisation. On the other hand, each approximating function is required to contain the DMUs that determine each straight-line segment. At the DMU with the smallest input and at that of the highest output, which, according to Ali (1993), are always efficient, it is only necessary to apply this constraint to a parabola, since these DMUs are the limits of the Pareto efficient frontier. For the others, the constraint must be applied to two parabolas, with $[2(n-2)+2]$ constraints being introduced.

Apart from these, the constraints of existence of derivatives (smoothness) must be considered, and they are necessary only in the intermediate DMUs, where the frontiers to the left and to the right are defined by distinct functions, $y_{i-1}$ and $y_{i}$. However, there are $n-2$ smoothness constraints and this results in the fact that the number of decision variables exceeds the number of constraints of equality by only one. Hence the problem is equivalent to the minimisation of a single-variable function, with the additional constraint of convexity.

\section{Formulation of the smoothing model for the two-dimensional DEA BCC problem}

Let $D$ be a set of DMUs, each with one input and one output. Further, let $E$ be the set of the Pareto efficient DMUs, $E_{l}, E_{2}, \ldots, E_{p}$, of input $x_{i}$ and output $y_{i}$. Further let us consider that the $E$ DMUs have their indices in rising order of input values. In this case the frontier-smoothing problem is represented by (II), this being a Quadratic Programming Problem (QPP).

$$
\begin{aligned}
& \min \sum_{i=1}^{p-1 x_{i+1}} \int_{x_{i}}\left\{1+\left[\left(a_{i} x^{2}+b_{i} x+c_{i}\right)^{\prime}\right]^{2}\right\} d x \\
& \text { st } \\
& a_{1} x_{1}^{2}+b_{1} x_{1}+c_{1}=y_{1}\left(\text { for } \mathrm{DMU}_{1}\right) \\
& a_{p-1} x_{p}^{2}+b_{p-1} x_{p}+c_{p-1}=y_{p}\left(\text { for } \mathrm{DMU}_{p}\right) \\
& a_{i-1} x_{i}^{2}+b_{i-1} x_{i}+c_{i-1}=y_{i}=a_{i} x_{i}^{2}+b_{i} x_{i}+c_{i}\left(\text { for } \mathrm{DMU}_{i}, \forall i \in(2, \ldots, p-1)\right) \\
& 2 a_{i-1} x_{i}+b_{i-1}=2 a_{i} x_{i}+b_{i}\left(\text { smooth frontier for } \mathrm{DMU}_{i}, \forall i \in(2, \ldots, p-1)\right) \\
& a_{i} \leq 0 \text { (frontier convexity) }
\end{aligned}
$$


The function shown in (II) minimises the sum of the arc lengths of the approximating functions $y_{i}=a_{i} x^{2}+b_{i} x+c_{i}$. The first two constraints ensure that the new frontier passes through the DMUs of smallest input and largest output, which mark the beginning of the Pareto inefficient region. In case there are reasons to consider that these DMUs should not be considered efficient by default, the corresponding constraints can be relaxed. The following constraints ensure continuity of the frontier and of its derivatives at the extreme efficient DMUs. The last constraint ensures convexity of the frontier.

This is a QPP. Although in must cases a solution can be found (in most of them in an analytical way), we will present and discuss a case of unfeasiblity.

\subsection{Example for the two-dimensional BCC case}

Table 1 shows values from an hypothetical example with one input and one output. The values in this table include the DMU with the smallest input at the origin, with the intent of reducing the complexity of the calculations. In a real case in which this DMU does not exist, a prior translation of the axes is necessary, which, although it alters the efficiencies, does not alter the geometry of the Pareto efficient frontier. Figure 5 shows the representation of this frontier.

We may see that the frontier is made up of DMUs A, B and D. If an input orientation were adopted, all the other DMUs would be projected onto the Pareto efficient region. If this were output oriented the DMU would be projected into a Pareto inefficient region. This region is the source of common DEA problems, which may be dealt with by methods derived from the smoothing model proposed in this paper (addition of constraints to the QPP).

Table 1 - Values of the numerical example for the BCC case with one input and one output.

\begin{tabular}{ccc}
\hline DMU & Input & Output \\
\hline A & 0 & 0 \\
B & 5 & 5 \\
C & 3 & 2 \\
D & 7 & 6 \\
E & 6 & 3 \\
F & 8 & 5 \\
\hline
\end{tabular}

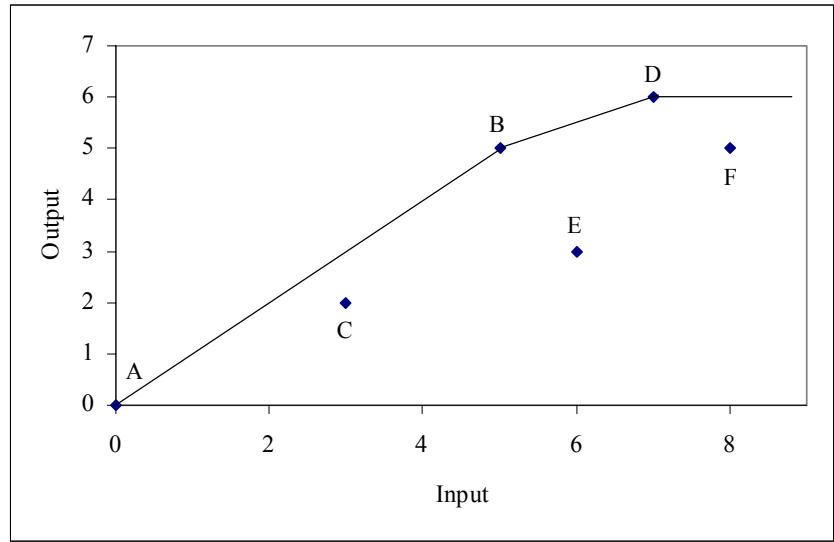

Figure 5 - Representation of the frontier in the numerical example. 
For this set of values two approximating functions are required, $y_{1}=a x^{2}+b x$ and $y_{2}=c x^{2}+d x+e$, and the frontier smoothing problem is given by (III).

$$
\begin{aligned}
& \min \left\{\int_{0}^{5}\left\{1+\left[\left(a x^{2}+b x\right)^{\prime}\right]^{2}\right\} d x+\int_{5}^{7}\left\{1+\left[\left(c x^{2}+d x+e\right)^{\prime}\right]^{2}\right\} d x\right\} \\
& =\min 5\left(1+b^{2}\right)+50 a b+500 / 3 a^{2}+2\left(1+d^{2}\right)+48 c d+872 / 3 c^{2}
\end{aligned}
$$

st

$25 a+5 b=5$

$25 c+5 d+e=5$

$49 c+7 d+e=6$

$10 a+b=10 c+d$

$a \leq 0$

$c \leq 0$

The solution of this QPP, solved analytically, is $\left\{\begin{array}{l}a=-1 / 35 \\ b=8 / 7 \\ c=-5 / 28 \\ d=37 / 14 \\ e=-15 / 4\end{array}\right.$, that provides the two approximating functions $y_{1}=-1 / 35^{2}+8 / 7 x$, valid in the stretch of the frontier defined by DMUs $\mathrm{A}$ and $\mathrm{B}$, and $y_{2}=-5 / 28 x^{2}+37 / 14 x-15 / 4$, valid for the efficient region of the frontier found between the DMUs B and D. The latter parabola can be extrapolated as far as part of the Pareto inefficient frontier.

Looking at Figure 6, which shows the smoothed frontier, we conclude that DMUs C and E are projected on to parabola $y_{1}$, when input orientation is adopted. For DMU $\mathrm{C}$ the projection point is $\mathrm{C}^{\prime \prime}$. That point is closer to the input axe than the original $\mathrm{BCC}$ projection point $\mathrm{C}^{\prime}$. On the other hand, $\mathrm{C}^{\prime \prime}$ is not so close to $\mathrm{C}$ as $\mathrm{C}^{\prime}$, meaning that $\frac{\mathrm{C}^{\prime} \mathrm{C}^{*}}{\mathrm{CC}^{*}}>\frac{\mathrm{C}^{\prime \prime} \mathrm{C}^{*}}{\mathrm{CC}^{*}}$. It follows that, in what concerns the efficiency value, the smoothed model is not so benevolent to the DMUs as the BCC one.

DMU F, still following input orientation, is projected onto the efficient DMU B, that is, simultaneously on to parabolas $y_{1}$ and $y_{2}$. Since there now exists a straight-line tangent to the frontier at DMU B, whose equation is $y-5=\frac{6}{7}(x-5)$, it is possible to determine the trade-off between this point's inputs and outputs, which is $6 / 7$. 


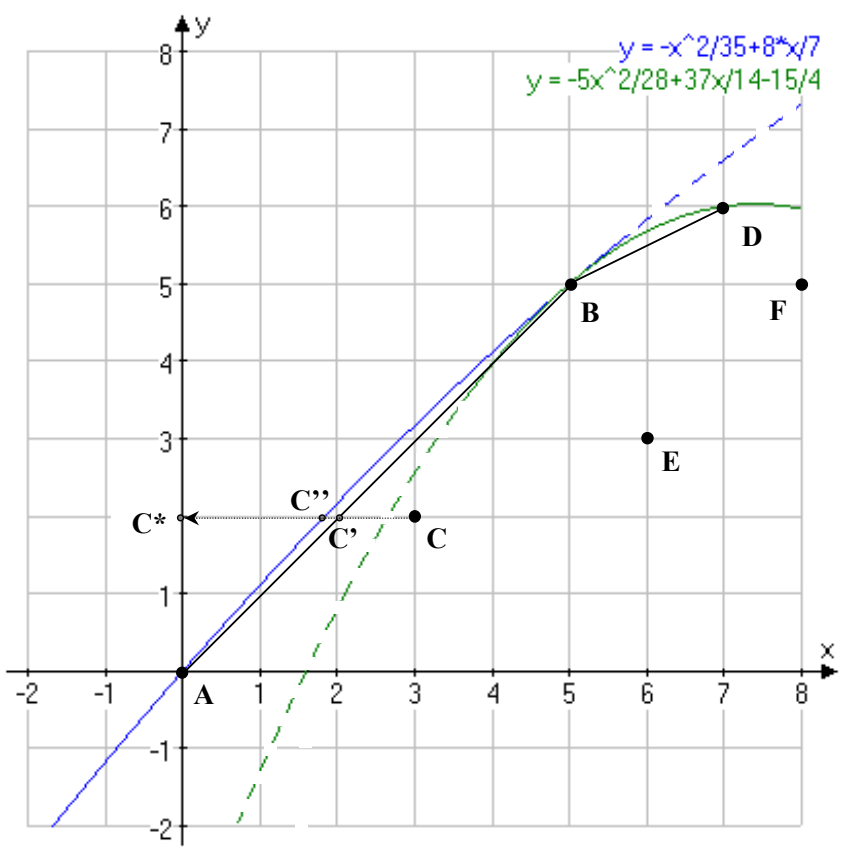

Figure 6 - Smoothed frontier in the numerical example.

When output orientation is used, DMU C continues to be projected onto parabola $y_{1}$, and DMU E becomes projected on to parabola $y_{2}$. Since the straight-line tangent to any point of parabola $y_{2}$ has angular coefficient $m=-5 / 14 x+37 / 14$, the output orientation shows a trade-off between inputs and outputs of $1 / 2$ for DMU E.

DMU F, still following output orientation, cannot be projected on to the calculated smoothed frontier. This is caused by parabola $y_{2}$ having its maximum for an input value $(x=7.4)$, less than the input of DMU F $(x=8)$. This means that DMU F would be being projected on to a decreasing region of the frontier, and that is not allowed. To solve this problem, additional constraints should be added. This must assume that the Pareto inefficient region starts at the DMU with the greatest input, and not at the efficient DMU with the greatest input. Thus, in this example, suffice it to impose the additional constraint $\left.\frac{\partial O}{\partial I}\right|_{F}=0$, generating a new smoothing model.

The frontier presented in this paper intersects the DEA frontier in the Pareto inefficient region. In the model with the additional constraint, the smoothed frontier will touch the BCC one in a region where no DMU is project, i.e., a region with no practical use. This model provides a smoothed frontier that, in a practical way, does not intersects the original one.

\subsection{QPP unfeasibility}

In the case of the frontier having more than one non-extreme-efficient DMU, unfeasibility of the smoothing QPP may occur. Table 2 shows values from a hypothetical example with one input and one output, that illustrate this situation. The values in this table are similar to those 
of Table 1, with the inclusion of DMUs $\mathrm{G}$ and $\mathrm{H}$, located on the efficient frontier, but not extreme-efficient. Figure 7 shows the representation of the efficient frontier, which is impossible to be smoothed.

Table 2 - Values of the numerical example for the BCC case with one input and one output.

\begin{tabular}{ccc}
\hline DMU & Input & Output \\
\hline A & 0 & 0 \\
B & 5 & 5 \\
C & 3 & 2 \\
D & 7 & 6 \\
E & 6 & 3 \\
F & 8 & 5 \\
G & 3 & 3 \\
H & 9 & 7 \\
\hline
\end{tabular}

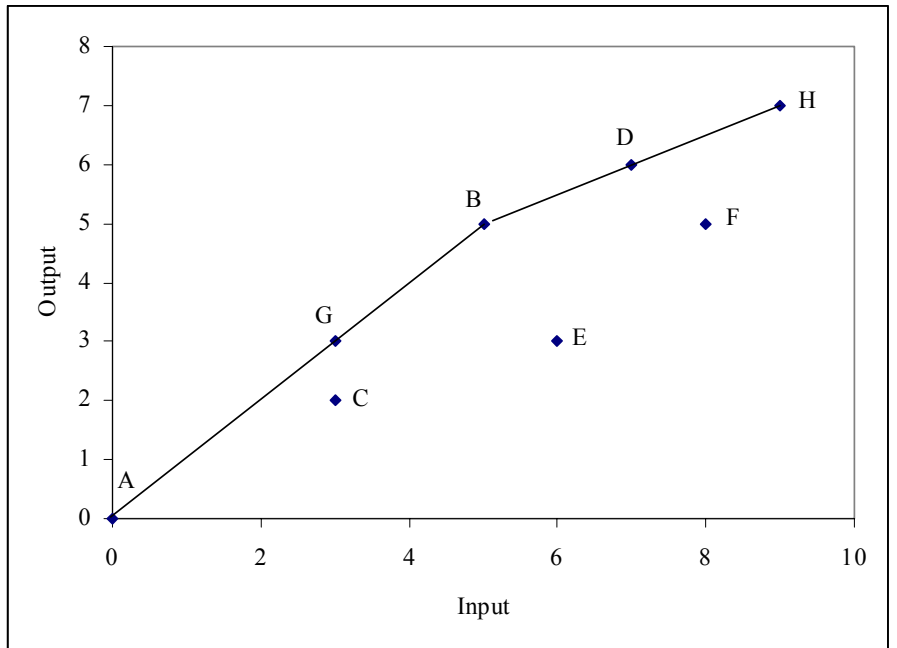

Figure 7 - Impossible frontier to be smoothed.

The inability for smoothing stems from the fact that the existence of three collinear points annuls the coefficient of the parabola's quadratic term.

If DMU $\mathrm{H}$ moves towards an infinitesimal reduction of its output, we have a first order contact problem between a straight line and a parabola (Pressley, 2001). This problem is always feasible. So, it is possible to build a Cauchy sequence of smoothable frontiers that converge to a non-smoothable frontier, meaning that the set of smoothable frontiers is a noncomplete metric set.

An approach for the infeasible case consists in relaxing the constraints that impose the smoothed frontier to contain all the efficient DMUs. We impose the smoothed frontier to be over the efficient DMUs, but that the greatest output difference must be as small as possible. 
The frontier of the Table 2 numerical example can be smoothed with a MixMax problem in (IV), already in the linear formulation. This LPP solution is showed in Table 3, where by the slacks analysis we can verify that DMUs A, B and $\mathrm{H}$ (extreme efficient DMUs) remain in the smoothed frontier. The efficient DMUs that are not in the smoothed frontier, are the nonextreme efficient ones.

$$
\begin{aligned}
& \min h \\
& \text { st } \\
& c \geq 0 \\
& 25 a+5 b+c \geq 5 \\
& 25 d+5 e+f \geq 5 \\
& 49 d+7 e+f \geq 6 \\
& 9 a+3 b+c \geq 3 \\
& 81 d+9 e+f \geq 7 \\
& 10 a+b=10 c+d \\
& h-c \geq 0 \\
& h-25 a-5 b-c+5 \geq 0 \\
& h-49 d-7 e-f+6 \geq 0 \\
& h-9 a-3 b-c+3 \geq 0 \\
& h-81 d-9 e-f+7 \geq 0 \\
& a \leq 0 \\
& d \leq 0
\end{aligned}
$$

Table 3 - MinMax problem solution.

\begin{tabular}{cc}
\hline Variable & Value \\
\hline$a$ & -0.0455 \\
$b$ & 1.2273 \\
$c$ & 0.0000 \\
$d$ & -0.0682 \\
$e$ & 1.4545 \\
$f$ & -0.5682 \\
surplus in DMU A & 0 \\
surplus in DMU B & 0 \\
surplus in DMU D & 0.2727 \\
surplus in DMU G & 0.2727 \\
surplus in DMU H & 0 \\
\hline
\end{tabular}

The parabolas equations are:

$$
y_{1}=-0.0455 x^{2}+1.2273 x \text { and } y_{2}=-0.0682 x^{2}+1.4545 x-0.5682 .
$$


The presence of efficient, but not extreme-efficient DMUs forces the smoothed frontier to be less closely to the original one. This effect is a consequence of using a MinMax objective function instead of minimising the arc length. To properly quantify this effect we need to measure the distance between the two frontiers: the original one and the smoothed frontier. Since an arc length based topology was used in the general formulation, we can use the arc length to measure the distance from the smoothed frontier to the original one. In order to avoid some technical difficulties in integral calculus, a similar function will be used.

Let the equation of the smoothed frontier be $y=f(x)$, and let the original DEA frontier be

represented by $y=h(x)$. The pseudo-metric $d=\frac{\int_{x_{1}}^{x_{n}}\left[f^{\prime}(x)\right]^{2} d x-\int_{x_{1}}^{x_{n}}\left[g^{\prime}(x)\right]^{2} d x}{\int_{x_{1}}^{x_{n}}\left[g^{\prime}(x)\right]^{2} d x}$ represents how

much the smoothed frontier is far from the DEA BCC original border. In the above equation $x_{1}$ is the smallest input a Pareto efficient DMU. The largest input of a Pareto efficient DMU is $x_{\mathrm{n}}$.

For the examples above, we have $d=0.00680$ in the case when the QPP has solution, and $d=0.14223$ in the situation when the QPP has no solution. This numbers clearly illustrate the effect of efficient but non-extreme-efficient DMUs presence.

\subsection{Smoothed model weights and cross-evaluation}

Once the smoothed frontier is known, the equation $u_{o} y-v_{o} x=u_{*}$ provides the smoothed model weights, $u_{o}, v_{o}$ and $u_{*}$. Since we have three variables and only one equation, we need yet two equations. They are obtained using the formulas for the parabola tangent. Combining these equations with the DEA BCC model equations we obtain (V).

$$
\begin{aligned}
& u_{o}=\frac{1}{x_{o}\left(2 a x_{o}+b\right)} \\
& v_{o}=\frac{1}{x_{o}} \\
& u_{*}=\frac{y_{o}}{x_{o}\left(2 a x_{o}+b\right)}-1
\end{aligned}
$$

In order to use the equations in $(\mathrm{V})$, inputs must be strictly positive.

Knowing the multipliers allows to solve some classical DEA problems. For instance, we will be able to know the exact return to scale of every DMU. In multi-dimensional problems, the importance of each input (or output) for every DMU can be determined.

The smoothed frontier also provides a new approach for the cross evaluation model (Doyle \& Green, 1995). It consists to evaluate one DMU by all the others. In order to perform this evaluation we need to calculate the arithmetic average of all the efficiencies obtained for a single DMU with the weights of the others. The smoothed model allows the weights to be uniquely determined, avoiding the choice of extreme models, such as the aggressive and benevolent ones. 


\subsubsection{Cross-evaluation example}

Table 4, obtained from Table 1 adding one unit to the inputs, is used for the cross-evaluation example.

Table 4 - Numerical data for the cross-evaluation.

\begin{tabular}{ccc}
\hline DMU & Input & Output \\
\hline A & 1 & 1 \\
B & 6 & 6 \\
C & 4 & 3 \\
D & 8 & 7 \\
E & 7 & 4 \\
F & 9 & 6 \\
\hline
\end{tabular}

Using equation (V) we obtain the weights in the smoothed model (Table 5).

Table 5 - Weights in the smoothed model for the efficient DMUs.

\begin{tabular}{cccc}
\hline DMU & $\boldsymbol{u}_{\boldsymbol{o}}$ & $\boldsymbol{v}_{\boldsymbol{o}}$ & $\boldsymbol{u}_{*}$ \\
\hline $\mathrm{A}$ & $7 / 8$ & 1 & $-1 / 8$ \\
$\mathrm{~B}$ & $7 / 36$ & $1 / 6$ & $1 / 6$ \\
$\mathrm{D}$ & $7 / 8$ & $1 / 8$ & $41 / 8$ \\
\hline
\end{tabular}

In Table 6 we can see the efficiency of all DMUs when evaluated by the efficient DMUs. We can observe the existence of negative efficiencies, not allowed by DEA general theory. This phenomena can only appear in BCC model when a DMU has an increasing return to scale.

Table 6 - Efficiency of all DMUs when evaluated by the efficient DMUs.

\begin{tabular}{cccc}
\hline \multirow{2}{*}{ DMU } & \multicolumn{3}{c}{ Evaluated by } \\
\cline { 2 - 4 } & A & B & D \\
\hline A & 1.000 & 0.167 & -34.000 \\
B & 0.896 & 1.000 & 0.167 \\
C & 0.688 & 0.625 & -5.000 \\
D & 0.781 & 0.896 & 1.000 \\
E & 0.518 & 0.524 & -1.857 \\
F & 0.597 & 0.667 & 0.111 \\
\hline
\end{tabular}

We can consider that DMU D, which leads to negative efficiencies, is not truly efficient and will not be allowed to evaluate other DMUs in the cross-evaluation model. In Table 7 we can see the average "cross-efficiencies". 
Table 7 - Average "cross-efficiencies".

\begin{tabular}{cc}
\hline DMU & Efficiencies \\
\hline B & 0.947917 \\
D & 0.838542 \\
C & 0.656250 \\
F & 0.631944 \\
A & 0.583333 \\
E & 0.520833 \\
\hline
\end{tabular}

\section{Conclusions}

Clearly the strong point of DEA is the study of cases with multiple inputs and outputs. This paper deals only with the less important case of one input and one output case. However a general theory for the general case has been developed. The main difficulty in solving the multi-dimensional case is the determinations of the facets equations. This is a NP hard problem (Fukuda, 1993). Another problem is that each extreme-efficient DMU belongs to an undetermined number of facets, avoiding to build the general smooth constraint. The multi-dimensional case needs a rather different approach, even using the same general theory. It may be possible to choose one single approximating function for the complete frontier, instead of one approximating function for every facet. Such an approach, for the special case of a single output and multi-outputs, has been presented by Soares de Mello et al. (2002).

The calculation of tradeoffs for the two-dimensional case leads to the definition of a new production possibilities set $P$. This new set is made up of the union of the set defined by the classic BCC model and the region of $\Re^{2}$ between the classic frontier and the parabolic arc which replaces it. Re-definitions of the set $P$ are common in the historical developments of DEA, but normally in the sense of constraining the production possibilities, as occurred when moving from the CCR model to the BCC model, and on to the FDH (Free Disposal Hull) model (Deprins et al., 1984). In all these cases, the changing of the set $P$ was beneficial to the DMUs, that is, caused increases in their efficiencies. In the case of smoothing of the frontier, the broadening of the set $P$ is more constraining on the DMUs, causing decreases in their efficiencies.

In the classic BCC model, all the DMUs belonging to the same facet present the same return to scale. Changes in the return to scale occur only at the vertices. In the smoothing model the return to scale varies continuously along the whole frontier.

This smoothed model can bring a new ranking in which some old efficient DMUs are no longer in the frontier. However, the aim of this model is not the ranking, but questions related to the tradeoffs and weights. It has been used in a new cross-evaluation model but new developments are needed in order to properly deal with negative efficiencies. It is important to emphasise that the smoothed frontier is not responsible for the negative efficiencies: they are a consequence of the constraints in the BCC model. 


\section{References}

(1) Ali, A.I. (1993). Streamlined computation for data envelopment analysis. European Journal of Operational Research, 64, 61-67.

(2) Andersen, P. \& Petersen, N.C. (1993). A Procedure for Ranking Efficient Units in Data Envelopment Analysis. Management Science, 39(10), 1261-1264.

(3) Banker, R.D.; Charnes, A. \& Cooper, W.W. (1984). Some models for estimating technical scale inefficiencies in Data Envelopment Analysis. Management Science, 30(9), 1078-1092.

(4) Charnes, A.; Cooper, W.W. \& Thrall, R.M. (1988). Classifying and characterizing efficiencies in data envelopment analysis. Operations Research Letters, 5(3), 105-110.

(5) Charnes, A.; Cooper, W.W.; Golany, B.; Seiford, L. \& Stutz, J. (1985). Foundations of data envelopment analysis for Pareto-Koopmans efficient empirical production functions. Journal of Econometrics, 30, 91-107.

(6) Chilingerian, J.A. (1995). Evaluating physician efficiency in hospitals: a multivariate analysis of best practices. European Journal of Operational Research, 80, 548-574.

(7) Coelli, T.; Rao, D.S.P. \& Battese, G.E. (1998). An Introduction to Efficiency and Productivity Analysis. Kluwer Academic Publishers.

(8) Deprins, D.; Simar, L. \& Tulkens, H. (1984). Measuring labour efficiency in post offices. In: The performance of public enterprises: concepts and measurement [edited by M. Marchand, P. Pestieau \& H. Tulkens], North Holand, Amsterdan, 243-267.

(9) Doyle, J.R. \& Green, R.H. (1995). Cross-evaluation in DEA: Improving discrimination among DMU's. INFOR, 33(3), 205-222.

(10) Elsgolts, L. (1980). Differential Equations and the Calculus of Variations. Mir Publishers, Moscow.

(11) Farlow, S.J. (1993). Partial Differential Equations for Scientists and Engineers. Dover Books on Advanced Mathematics, Dover Publications.

(12) Fukuda, K. (1993). cdd.c: C Implementation of the Double Description method for computing all vertices and extremal rays of a convex polyhedron given a system of linear inequalities. Department of Mathematics, Swiss Federal Institute of Technology, Lausanne, Switzerland.

(13) Gal, T. (1992). Weakly redundant constraints and their impact on postoptimal analyses in LP. European Journal of Operational Research, 60(3), 315-326.

(14) Hausdorff, F. (1949). Grundzüge der Mengenlehre. Chelsea Publishing Company, New York.

(15) Koohyun, P. \& Yong-Sik, S. (1998). Iterative bundle-based decomposition for largescale nonseparable convex optimisation. European Journal of Operational Research, 111(3), 598-616.

(16) Lins, M.P.E. \& Angulo-Meza, L. (2000). Análise Envoltória de Dados e perspectivas de integração no ambiente de Apoio à Decisão. Editora da COPPE/UFRJ, Rio de Janeiro, Brazil. 
(17) Lipschutz, S. (1968). Schaum's Outline of General Topology. McGraw Hill.

(18) Mumem, M. (1984). Calculus With Analytic Geometry. Worth Publishing.

(19) Olesen, O.P. \& Petersen, N.C. (1996). Indicators of III conditioned data sets and model misspecification in data envelopment analysis: and extended facet approach. Management Science, 42(2), 205-219.

(20) Prenter, P.M. (1975). Splines and Variational Methods. John Wiley \& Sons, Inc., New York.

(21) Pressley, A. (2001). Elementary Differential Geometry. Springer.

(22) Reddy, J.N. (1993). Introduction to the Finite Element Method. McGraw-Hill.

(23) Rosen, D.; Schaffnit, C. \& Paradi, J.C. (1998). Marginal rates and two dimensional level curves in DEA. Journal of Productivity Analysis, 9(3), 205-232.

(24) Smith, D.R. (1998). Variational Methods in Optimization. Dover Books on Mathematics, Dover Publications.

(25) Soares de Mello, J.C.C.B. (1987). Métodos variacionais em condução de calor. M.Sc. Thesis, Fluminense Federal University, Niterói, Brazil.

(26) Soares de Mello, J.C.C.B.; Lins, M.P.E.; Gomes, E.G. \& Biondi, L.N. (2002). Suavização da fronteira DEA: O caso BCC tridimensional. Actas do IO2002 - X Congresso da Associação Portuguesa de Investigação Operacional, Guimarães, Portugal.

(27) Thanassoulis, E. (1993). A comparison of regression analysis and data envelopment analysis as alternative methods of performance assessment. Journal of the Operational Research Society, 44(11), 1129-1144. 